\title{
PROXIMITY: An update and expansion of the PROXTIME computer program
}

\author{
BONNIE N. POLLACK \\ University of North Carolina, Charlotte, North Carolina \\ and \\ BENJAMIN B. CHASE \\ Concord, North Carolina
}

\begin{abstract}
A new program, called PROXIMITY, was created as a tool for proximity calculation and to update and expand upon Kirste and Monge's (1983) PROXTIME program. The purpose of PROXIMITY is to calculate the fluctuating proximity between individuals within organizations. PROXIMITY provides output for three types of relationships: (1) an overall organizational proximity, (2) pairwise proximity between individuals, and (3) individual proximity to multiple others. PROXIMITY also updates some of PROXTIME's features such as the computer platform, the type of data the program can handle, and the form of output available.
\end{abstract}

Interpersonal proximity in organizations is an important factor in understanding a variety of outcomes in fields such as environmental planning, communication, social psychology, and organizational behavior. In environmental planning, researchers are interested in how proximity affects crowding (e.g., Baum \& Paulus, 1987; Griffitt \& Veitch, 1971; Moos, 1976), privacy (e.g., Sundstrom, 1987), and stress (e.g., Evans \& Cohen, 1987), as well as human comfort, territoriality, and spatial planning (e.g., Altman, 1975; Sundstrom, 1986). Communication researchers are interested in how proximity affects general communication patterns (e.g., Smith \& Kearny, 1994), preferences for amount and recipient of social interactions (e.g., Zahn, 1991), and type of communication (workrelated versus casual/informal) (e.g., Becker \& Steele, 1995; Steele, 1986). Researchers in social psychology are interested in how proximity affects group idea generation (e.g., Valacich, George, Nunamaker, \& Vogel, 1994), and interpersonal attraction (e.g., Arkin \& Burger, 1980). Organizational behavior researchers are interested in how proximity affects attitudes about co-workers (e.g., Moos, 1976), organizational and job satisfaction (e.g., Oldham \& Fried, 1987), and task performance (e.g., Becker \& Steele, 1995; Smith \& Kearny, 1994).

Though proximity can be operationalized in many ways (e.g., linear distance, functional distance, and psychological distance), one of the most useful operationalizations

This research was partially funded by a grant to the first author from the University of North Carolina at Charlotte Junior Faculty Summer Fellowship Program. We thank Matt Kurbat, Sarah Breedin, and Paula Goolkasian for their assistance in completing this publication. A copy of the program and additional user-oriented documentation can be obtained at no cost, except for postage and inclusion of a disk, from B. N. Pollack, 12 15th Avenue, San Francisco, CA 94118 (e-mail: bonnienp@ hotmail.com). of interpersonal proximity in organizations was developed by Monge and Kirste (1980), which they described as "fluctuating distance." This operationalization of proximity improves upon earlier measures because it captures the dynamic nature of people's movement over time, and recognizes that individuals are concurrently proximate to many others in an organizational setting. In 1983, Kirste and Monge introduced a computer program, called PROXTIME, to calculate fluctuating proximity between individuals within organizations. Fluctuating proximity is "the probability of people being in the same "communication location' during the same interval of time" (Monge \& Kirste, 1980, p. 112).

Kirste and Monge's (1983) PROXTIME program was a good initial step toward calculating fluctuating proximity, and some research has made use of it (e.g., Monge \& Kirste, 1980, using an earlier version called PROXVAL; Monge, Rothman, Eisenberg, Miller, \& Kirste, 1985). We wanted to create a proximity calculation program for the current computing environment that also expanded upon PROXTIME's capabilities. Thus, we created a new program, called PROXIMITY. Below, we discuss some of the features of PROXTIME. After each feature, we explain the modifications that we have made and incorporated into our new PROXIMITY program. They include (1) the computer platform used, (2) the unit of time for the output, (3) the addition of a third proximity calculation to the original two, (4) the form of the input data, (5) the way in which the work week is defined, and (6) the choice of calculations for the three different types of proximity.

The first thing that we modified in creating this new program was the platform (and thus the computer-user interface as well). Probably the greatest limitation for current computer users is that PROXTIME was written in the FORTRAN computer language and must be used with a mainframe computing system that supports a FOR- 
TRAN IV compiler. PROXIMITY is a user-friendly desktop program (i.e., no understanding of computer languages is needed for one to use it) that operates on Macintosh computers. The program has also been ported to a UNIX system (Solaris 2.5.1) and can be used on this platform as well.

The second modification we made was to change the program's output from dozens of individual results in time units of 15 min each to single summary results averaged together from part of a day (minutes or hours) to a full work week. Although a time unit as fine grained as 15 min can be interesting in some types of research (e.g., Monge et al., 1985), many organizational researchers are interested in results across much larger units of time, such as full days or entire work weeks (e.g., Kirste \& Monge, 1983; Monge \& Kirste, 1980; Zahn, 1991). PROXIMITY allows the user to determine what block of time is most useful and provides output for various proximity measures, ranging from those as fined grained as $1 \mathrm{~min}$ to a summary for the entire work week.

The third change that we made was to add the computation of a third type of proximity. PROXTIME calculates pairwise or dyad-level proximity (an individual's proximity with each other person in the organization) and proximity to multiple others (an individual's proximity to all others in the organization). Another useful type of proximity is what Kirste and Monge $(1983$, p. 89$)$ and Monge et al. (1985, p. 1129) described as "organizational proximity" (the degree to which people within an organization share the same physical locations during the same periods of time). PROXTIME does not compute its value, so in addition to pairwise proximity and proximity to multiple others, we programmed PROXIMITY to calculate this overall organizational proximity. Mathematical definitions of these three types of proximity are provided below.

The fourth modification concerns the format of the input data. In PROXTIME, specifications for how the data have to be entered are fairly rigid (see Kirste \& Monge, 1983 , for details). PROXIMITY has the following new features not present in PROXTIME: Data are now taken from a spreadsheet; individuals and locations can be given full names; the length of time intervals can be determined by the user; all 7 days of the week are acceptable for input; start and end times for the work days can be determined by the user; the length of the work week is automatically determined by the given start and end days and times.

Fifth, in the new program we give the user more control over how the work week is defined. In PROXTIME, the work week could only be $50 \mathrm{~h}$, only Monday through Friday, only 8 a.m. to 6 p.m. PROXIMITY, on the other hand, allows the user to pick any choice of start and end days and times. This enables the researcher to select a work week that is meaningful in the situation under study.

Sixth, in the new program we give the user more choices for calculating each of the three different types of proximity. For the overall organizational proximity and the proximity to multiple others values, the user can allow the length of the work week to be determined by start and end days and times, or by the length of time when at least one person was present at the site. For the pairwise proximity values, the user can allow the length of the work week to be determined by start and end days and times, or by the amount of overlapping hours between the two people in the dyad (i.e., the hours in which they were both present at the site). These choices allow the researcher to tailor the proximity measures to the research question under study.

\section{PROXIMITY}

General description. PROXIMITY calculates (1) organizational proximity, which is the degree to which people within an organization share the same physical locations during the same periods of time for the entire work week; (2) pairwise proximity, which is an individual's proximity to each other individual in the organization (dyad-level information) for the entire work week; and (3) proximity to multiple others, which is an individual's proximity to all other individuals in the organization for the entire work week. PROXIMITY can handle an unlimited number of individuals at 2 -min or greater time intervals over a 7-day, and up to a 24 -h/day work week.

Structure of input data for PROXIMITY runs. PROXIMITY takes a spreadsheet or tab-delimited lines as input. An example spreadsheet is given in Table 1. This spreadsheet needs to be five contiguous columns wide by some number of contiguous rows long. No heading should be included. The columns should contain the data in the following order: person's name, day, room name, start time, and end time. Individuals and room locations can be written as names or numbers. The day field is an integer in the range 0 to 6 (i.e., Sunday to Saturday). The start and end time is in an HHMM 24-h/military format (i.e., $\mathrm{HH}$ varies from 0 to 23 and $\mathrm{MM}$ varies from 00 to 59). Start and end times cannot overlap; in other words, a person cannot be recorded as occupying two different rooms at the same time. So room occupa-

Table 1

Sections of an Example Spreadsheet for PROXIMITY Input Data

$\begin{array}{lllll}\text { Adam } & 1 & \text { Room } 1 & 0730 & 0859 \\ \text { Adam } & 1 & \text { Room2 } & 0900 & 0929 \\ \cdot & & & & \\ \cdot & & & & \\ \text { Beth } & 1 & \text { Room2 } & 0800 & 1129 \\ \text { Beth } & 1 & \text { Room4 } & 1130 & 1229 \\ \cdot & & & & \\ \cdot & & & & \\ \text { Charlie } & 1 & \text { Room4 } & 0800 & 0829 \\ \text { Charlie } & 1 & \text { Room3 } & 0830 & 1129 \\ & & & & \end{array}$


tion data should be recorded, for example, as a person being in Room 1 from 9:00 to 9:29 a.m., and then Room 2 from 9:30 to 9:59 a.m., instead of Room 1 from 9:00 to 9:30 a.m. and Room 2 from 9:30 to 10:00 a.m. Each row contains information for, within a particular day, what room a person occupies between which times.

Error checking. Extensive error checking is automatically conducted throughout the program, with the user having the option of viewing it. At the beginning of the program run, the user has the option of specifying choices of "normal output," "verbose output," or "very verbose output." Each choice respectively gives the user more information. For all three output choices, the program provides basic information about the data so that the user can examine it and verify its accuracy. When PROXIMITY detects an error, it will print out an appropriate error message to guide the user. If the user enters an invalid choice in response to a question, the program will prompt the user to try again.

Limitations. Unlike PROXTIME, PROXIMITY has no limitations on the number of individual identifiers (i.e., number of people) or location/time combinations. It does have some other limitations, though. Like PROXTIME, it can only process data for a maximum of 1 work week ( 7 days), and the time must be specified in military time. The unit of time is minutes, and the smallest time intervals that the program will accept are $2 \mathrm{~min}$ long. People and location names are limited in length to 100 characters. Unlike PROXTIME, PROXIMITY will allow data for 24-h days and workshifts that go around the clock. In this case, the end time must be specified as 23:59 of one day, and the next start time must be specified as 00:00 of the next day, with data from each of the days placed on separate lines on the Excel spreadsheet.

Output. The program's output is captured in the user's input file (i.e., all input and output information is contained in the same scrolling text window), so the user has complete information about a particular run in a single file. First, the program displays some descriptive statistics about the input data, so that the user may verify its accuracy. Then the three proximity values are calculated and displayed. At the end of the run, the user may print the entire file, save it as a text file, and/or cut and paste it into a word processing program. As a saved file, the user can access it within a word processing program.

Calculations. The program calculates three different kinds of proximity values. Each of these kinds is calculated at specific times during the work week and then averaged over these times. The second and third type described below are the same as Kirste and Monge's (1983), except that the results are for the entire work week. The first type described below is our added proximity, for the entire organization. An example of sample output is given in Table 2.

Before any proximity calculations are made, the program reads the data in samples. The user specifies the length of the sample intervals, which can be as fine grained as the intervals at which the data were collected (to a min-
Table 2

Sections of Example PROXIMITY Output for the Three Types of Proximity

Group Proximity is 0.0337 .

Value used for group proximity denominator was 132 .

The dyads shown below are for certain pairs of people P1 and P2. Because the value should be the same for the pair $\mathrm{P} 1, \mathrm{P} 2$ and the pair $\mathrm{P} 2, \mathrm{P} 1$, only one of these two pairs is shown.

$\begin{array}{lll}\text { P1 } & \text { P2 } & \text { Dyad Proximity Value } \\ \text { Adam } & \text { Beth } & 0.0379 \\ \text { Adam } & \text { Charlie } & 0.0000 \\ \text { Beth } & \text { Charlie } & 0.0379\end{array}$

The value printed below, next to each person, is that person's proximity to all others. This is also called "proximity to multiple others." The denominator used for these is 132 .

\begin{tabular}{ll} 
Person & Proximity to Multiple Others \\
Adam & 0.0168 \\
Beth & 0.0337 \\
Charlie & 0.0168 \\
\hline
\end{tabular}

imum of $2 \mathrm{~min}$ ). For each sample interval, the program reads the data once to see which room a particular person occupies. These are called samples, which are then used in the proximity calculations.

The first kind of proximity value is the proximity of the entire group of $N$ workers (i.e., the whole organization). At a given moment in time, the locations of all the workers are noted. For every location where there is more than one person, the number of persons is squared and then divided by the square of $N$, and all of these values are summed. The work week average is obtained by summing its value over all the sample times and then dividing by the number of samples. In Table 2 , the organizational proximity, labeled "Group Proximity," equals 0.0337 .

The second kind of proximity value is the pairwise or dyad-level proximity. At a given moment in time, for a particular pair of workers, if they are in the same location, their pairwise proximity is 1 ; otherwise, it is 0 . This value is calculated for all pairs. The work week average is obtained by summing the values for a particular pair for some number of samples and then dividing by the number of samples. The user can choose the number of samples to be either for the entire work week, or just those samples when both workers were somewhere at work. In Table 2, the dyadic proximity values are found in the column labeled "Dyad Proximity Value."

The third kind of proximity value is the proximity to multiple others. At a given moment in time, for a particular worker, his/her proximity to multiple others is the square of the number of people in his/her location, divided by the square of $N$, unless he/she is either not at work or working alone, in which case his/her proximity to multiple others at that time is 0 . The values for a particular person are averaged over all the samples in the work week to obtain an average proximity to multiple 
others. In Table 2, the proximity to multiple others values are found in the column labeled "Proximity to Multiple Others."

Hardware and transportability. The program was compiled on a 68040 Macintosh and will operate on newer models of Macintosh. It was tested on a Macintosh Performa 636 running Operating System 7.5.3 and on a Power Macintosh 6100 running Operating System 7.5.1. The source code is written in ANSI C. The program was developed under CodeWarrier, and for the program to be modified, CodeWarrier is needed. To simply run the program, the user does not need the authoring program; the program is a self-contained, stand-alone application. The program can be used on UNIX systems.

Program availability. A copy of the program and additional user-oriented documentation can be obtained at no cost, except for that of postage and the inclusion of a disk, from the first author at 12 5th Avenue, San Francisco, CA 94118.

\section{REFERENCES}

Altman, I. (1975). The environment and social behavior. Monterey, CA: Brooks/Cole.

ARKIN, R. M., \& BURGER, J. M. (1980). Effects of unit relation tendencies on interpersonal attraction. Social Psychology Quarterly, 43, 380-391.

Baum, A., \& Paulus, P. B. (1987). Crowding. In D. Stokols \& I. Altman (Eds.), Handbook of environmental psychology (pp. 533-570). New York: Wiley.

BeCKer, F., \& STEELE, F. (1995). Workplace by design: Mapping the high-performance workscape. San Francisco: Jossey-Bass.

Evans, G. W., \& Cohen, S. (1987). Environmental stress. In D. Stokols \& I. Altman (Eds.), Handbook of environmental psychology (pp. 571610). New York: Wiley.
GriffitT, W., \& VeITCH, R. (1971). Hot and crowded: Influences of population density and temperature on interpersonal affective behavior. Journal of Personality \& Social Psychology, 17, 92-98.

KiRSTE, K. K., \& MONGE, P. R. (1983). Computing dynamic organizational proximity: The PROXTIME computer program. Behavior Research Methods \& Instrumentation, 15, 89-90.

MONGE, P. R., \& KIRSTE, K. K. (1980). Measuring proximity in human organization. Social Psychology Quarterly, 43, 110-115.

Monge, P. R., Rothman, L. W., EisenberG, E. M., Miller, K. I., \& KIRSTE, K. K. (1985). The dynamics of organizational proximity. Management Science, 31, 1129-1141.

Moos, R. H. (1976). The human context: Environmental determinants of behavior. New York: Wiley.

Oldham, G. R., \& FrIED, Y. (1987). Employee reactions to workspace characteristics. Journal of Applied Psychology, 72, 75-80.

SMITH, P., \& KeARNY, L. (1994). Creating workplaces where people can think. San Francisco: Jossey-Bass.

STEELE, F. I. (1986). Making and managing high-quality workplaces: An organizational ecology. New York: Columbia University, Teachers College Press.

Sundstrom, E. (1986). Work places: The psychology of the physical environment in offices and factories. Cambridge: Cambridge University Press.

Sundstrom, E. (1987). Work environments: Offices and factories. In D. Stokols \& I. Altman (Eds.), Handbook of environmental psychology (pp. 733-782). New York: Wiley.

Valacich, J. S., George, J. F., Nunamaker, J. F., \& Vogel, D. R. (1994). Physical proximity effects on computer-mediated group idea generation. Small Group Research, 25, 83-104.

ZAHN, G. L. (1991). Face-to-face communication in an office setting: The effects of position, proximity, and exposure. Communication Research, 18, 737-754.

(Manuscript received November 15, 1999; revision accepted for publication March 3, 2000.) 